International Journal of Pure and Applied Mathematics

Volume 104 No. 2 2015, 237-247

ISSN: 1311-8080 (printed version); ISSN: 1314-3395 (on-line version)

url: http://www.ijpam.eu

doi: http://dx.doi.org/10.12732/ijpam.v104i2.8

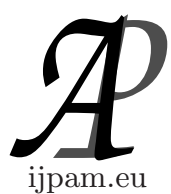

\title{
SOFT TOPOLOGICAL QUESTIONS AND ANSWERS
}

\author{
M. Matejdes \\ Department of Mathematics and Computer Science \\ Faculty of Education, Trnava University in Trnava \\ Priemyselná 4, 91843 Trnava, SLOVAKIA
}

\begin{abstract}
The paper deals with a few questions concerning a soft topological space. The main goal is to point out that any soft topological space is homeomorphic to a topological space $\left(A \times X, \tau_{A \times X}\right)$ where $\tau_{A \times X}$ is a topology on the product $A \times X$, consequently many soft topological notions and results can be derived from general topology.
\end{abstract}

AMS Subject Classification: 54C60, 26A15, 26E25

Key Words: soft set, soft topological space, soft closure, $\theta$-closure, separation axioms, soft $e$-continuity, soft $e-\theta$-continuity

\section{Introduction}

The recent interest (see the references) in the soft topological spaces is growing and intensive study contributes both to the development of the soft set theory, but also brings many open problems.

In [5], for a soft topological space $(A, X, \tau$,$) , the next propositions were$ proved (for the definitions and notations see [5]) and the authors ask if the converses of Propositions 3.11, 3.16, 3.17, 5.33 below are true as well as they ask to find a connection between two soft topologies $\tau$ and $\tau_{\theta}$.

Received: July 14, 2015

(c) 2015 Academic Publications, Ltd. url: www.acadpubl.eu 
(1) Proposition 3.11 If for every $x \in X$, for every $a \in A$ and for every $a$-soft open neighborhood $(F, A)$ of $x$ there exists an $a$-soft open neighborhood $(G, A)$ of $x$ such that $x \in G(a) \subset \operatorname{cl}(G, a) \subset F(a)$, then $(X, \tau, A)$ is a soft $T_{3}$-space.

(2) Proposition 3.16 Let $(F, A) \in S S(X, A)$ and $a \in A$. If there exists a net $S=\left\{x_{\lambda}, \lambda \in \Lambda\right\}$ of $X$ such that $x_{\lambda} \in_{a}(F, A)$, for every $\lambda \in \Lambda$ and $x \in$ s- $\lim (S)$, then $x \in \in_{a} c l(F, A)$.

(3) Proposition 3.17 Let $\left(A, X, \tau_{X}\right)$ and $\left(B, Y, \tau_{Y}\right)$ be two soft topological spaces, $x \in X$ and $e$ a map of $A$ onto $B$. If the map $f: X \rightarrow Y$ is soft $e$ continuous at the point $x$, then for every net $S=\left\{x_{\lambda}, \lambda \in \Lambda\right\}$ of $X$ which soft converges to $x$ in $\left(A, X, \tau_{X}\right)$ we have that the net $\left\{f\left(x_{\lambda}\right), \lambda \in \Lambda\right\}$ of $Y$ soft converges to $f(x)$ in $\left(B, Y, \tau_{Y}\right)$.

(4) Proposition 5.33 If the map $f: X \rightarrow Y$ is soft $e-\theta$-continuous, then $\Phi_{f e}^{-1}(G, B)$ is a soft subset of $\operatorname{int}_{\theta}\left(\Phi_{f e}^{-1}\left(c l_{\theta}(G, B)\right)\right.$ for every $(G, B) \in \tau_{Y}$.

(5) Under which conditions does the equality $\tau=\tau_{\theta}$ holds?

(6) Under what conditions does the sequence $\tau, \tau_{\theta},\left(\tau_{\theta}\right)_{\theta},\left(\left(\tau_{\theta}\right)_{\theta}\right)_{\theta}, \ldots$ is eventually constant?

(7) Find a soft topological space such that the sequence $\tau, \tau_{\theta},\left(\tau_{\theta}\right)_{\theta},\left(\left(\tau_{\theta}\right)_{\theta}\right)_{\theta}, \ldots$ is strictly decreasing?

\section{Topological and soft topological space}

Any subset $S$ of the Cartesian product $A \times X$ is called a relation from $A$ to $X$. By $\mathbf{R}(A, X)$, we denote the set of all binary relations from $A$ to $X$ and $S[a]:=\{x \in X:[a, x] \in S\}$. The operations of the sum $S \cup T, \cup_{t \in T} S_{t}$, the intersection $S \cap T, \cap_{t \in T} S_{t}$, the complement $S^{c}$ and the difference $S \backslash T$ of relations are defined obvious way as in the set theory.

By $F: A \rightarrow 2^{X}$ we denote a set valued mapping from $A$ to the power set $2^{X}$ of $X$. A set of all set valued mappings from $A$ to $2^{X}$ is denoted by $\mathbf{F}(A, X)$. If $F, G$ are two set valued mappings, then $F \subset G(F=G)$ means $F(a) \subset G(a)$ $(F(a)=G(a))$ for any $a \in A$.

A graph of a set valued mapping $F$ is the set $\operatorname{Gr}(F):=\{[a, x] \in A \times X$ : $x \in F(a)\}$ and it is a subset of $A \times X$, hence $\operatorname{Gr}(F) \in \mathbf{R}(A, X)$. So, any set 
valued mapping determines a relation from $\mathbf{R}(A, X)$ denoted by $R_{F}:=\{[a, x] \in$ $A \times X: x \in F(a)\}=G r(F)$.

On the other hand, any relation $S \in \mathbf{R}(A, X)$ determines a set valued mapping $F_{S}$ from $A$ to $2^{X}$ where $F_{S}(a)=S[a]$. So, there is one-to-one correspondence between the relations from $\mathbf{R}(A, X)$ and the set valued mappings from $\mathbf{F}(A, X)$, i.e., if $S \in \mathbf{R}(A, X)$ and $G, H \in \mathbf{F}(A, X)$, then

$$
\begin{gathered}
S \mapsto F_{S}, F_{S}(a)=S[a], \quad G \mapsto R_{G}, R_{G}[a]=G(a), \\
F_{R_{G}}=G, F_{R_{G}}(a)=G(a), R_{F_{S}}=S, R_{F_{S}}[a]=S[s], \\
H=F_{S} \Leftrightarrow R_{H}=S .
\end{gathered}
$$

Definition 2.1. For $H, G, F_{t} \in \mathbf{F}(A, X), t \in T$, we define the following obvious set valued mapping operations.

(1) Sum: $\cup_{t \in T} F_{t}: A \rightarrow 2^{X}, a \mapsto \cup_{t \in T} F_{t}(a)$,

(2) Intersection: $\cap_{t \in T} F_{t}: A \rightarrow 2^{X}, a \mapsto \cap_{t \in T} F_{t}(a)$,

(3) Complement: $H^{c}: A \rightarrow 2^{X}, a \mapsto X \backslash H(a)$,

(4) Difference: $H \backslash G: A \rightarrow 2^{X}, a \mapsto H(a) \backslash G(a), a \in A$.

In this section we will consider the soft sets over common an initial universe set $X$ and a fixed set of parameters $A$ and a definition of a soft set is introduced by a set valued mapping (see the references).

Definition 2.2. If $G: A \rightarrow 2^{X}$ is a set valued mapping, then a pair $(G, A)$ is called a soft set over $X$ with respect to a set of parameters $A$. The family of all soft sets over $X$ with respect to $A$ is denoted by $S S(A, X)$. A triplet $(A, X, \tau)$ where $\tau \subset S S(A, X)$ is a soft topology is called a soft topological space $([5])$.

As we said above there is no difference between a set valued mapping $F \in$ $\mathbf{F}(A, X)$ and its graph $\operatorname{Gr}(F)$, which is a member of $\mathbf{R}(A, X)$. So, a soft set can be defined as follows.

Definition 2.3. A soft set over $X$ with respect to $A$ is a pair $(S, A)$, where $S \subset A \times X$. So, in this case a soft set is represented by a relation from $\mathbf{R}(A, X)$. 
The basic soft operations (the soft union, the soft intersection, the soft complement) can be defined by the corresponding set valued mapping operations on $\mathbf{F}(A, X)$ stated in Definition 2.1 or by the corresponding relation operations. For example $\sqcup_{t \in T}\left(G_{t}, A\right)=\left(\cup_{t \in T} G_{t}, A\right)=\left(F_{\cup_{t \in T} R_{G_{t}}}, A\right)$.

From the one-to-one correspondence between the relations from $\mathbf{R}(A, X)$ and the set valued mappings from $\mathbf{F}(A, X)$, a soft topological space can be characterizes as follows.

Theorem 2.1. A triplet $(A, X, \tau)$ if a soft topological space if and only if $\left(A \times X, \tau_{A \times X}\right)$ is a topological space, where $\tau_{A \times X}=\left\{R_{G}:(G, A) \in \tau\right\}$.

By Definition 2.2, any member from $\tau$ (called a soft open set) is represented by a set valued mappings $G$ from $\mathbf{F}(A, X)$ which determines the binary relation $R_{G}$ (open set) from $\tau_{A \times X}=\left\{S \in \mathbf{R}(A, X):\left(F_{S}, A\right) \in \tau\right\}$ which forms a topology on $A \times X$.

On the other hand, if $\left(A \times X, \tau_{A \times X}\right)$ is a topological space with the topology $\tau_{A \times X} \subset \mathbf{R}(A, X)$, then $(A, X, \tau)$ is a soft topological space, where $\tau=\{(G, A) \in$ $\left.S S(A, X): R_{G} \in \tau_{A \times X}\right\}$. This observation can be expressed by the equivalences

$$
(G, A) \in \tau \Leftrightarrow R_{G} \in \tau_{A \times X}, \quad S \in \tau_{A, X} \Leftrightarrow\left(F_{S}, A\right) \in \tau .
$$

For $P=[a, x]$, a soft set $\left(G_{P}, A\right)$, where $G_{P}\left(a_{1}\right)=\{x\}$ for $a_{1}=a$ and $G_{P}\left(a_{1}\right)=\emptyset$ for $a_{1} \neq a$ is called a soft point. Denote by $S S_{0}(A, X)$ the set of all soft pints. Then the next theorem expresses a homeomorphic connection between $(A, X, \tau)$ and $\left(A \times X, \tau_{A \times X}\right)$.

Theorem 2.2. Any soft topological space $(A, X, \tau)$ is homeomorphic with a topological space $\left(A \times X, \tau_{A \times X}\right)$ and a homeomorphism $h:\left(S S_{0}(A, X), \tau\right) \rightarrow$ $\left(A \times X, \tau_{A \times X}\right)$ is given by $h\left(\left(G_{P}, A\right)\right)=[a, x]$, where $P=[a, x]$.

If $\left(A \times X, \tau_{A \times X}\right)$ is a topological space, then for any $a \in A, \tau_{A \times X}$ induces a topology $\tau_{\{a\} \times X}$ on $\{a\} \times X$. A topological subspace $\left(\{a\} \times X, \tau_{\{a\} \times X}\right)$ is denoted briefly by $\left(X, \tau_{a}\right)$. Generally, for any soft topological space $(A, X, \tau)$, we can define its soft topological subspace as the homeomorphic image of a topological subspace $\left(S, \tau_{S}\right)$ of the topological space $\left(A \times X, \tau_{A \times X}\right)$, where $S \subset A \times X$ and $\tau_{S}$ is the induced topology. In literature, a soft topological subspace is usually defined only for $Y \subset X$.

Recall the soft set $\mathbf{1}_{A}\left(=\left(F_{A \times X}, A\right)\right)$ and $\mathbf{0}_{A}\left(=\left(F_{\emptyset}, A\right)\right)$ are the absolute soft set and the null soft set, respectively. The correspondence between the 
soft open (soft closed) sets and the open (closed) sets can be formulated by the following lemma.

Theorem 2.3. A soft set $(G, A)$ is soft open (soft closed) in a soft topological space $(A, X, \tau)$ if and only if $R_{G}$ is open (closed) in the corresponding topological space $\left(A \times X, \tau_{A \times X}\right)$ and $S$ is open (closed) in a topological space $\left(A \times X, \tau_{A \times X}\right)$ if and only if $\left(F_{S}, A\right)$ is soft open (soft closed) in the corresponding soft topological space $(A, X, \tau)$.

The main result of this section shows a deep connection between a soft topology $\tau$ on $S S(A, X)$ and the corresponding topology $\tau_{A \times X}=\{S \subset A \times X$ : $\left.\left(F_{S}, A\right) \in \tau\right\}$ on the product $A \times X$. Any soft topological space $(A, X, \tau)$ can be considered as a topological space on $A \times X$ and any topological space $(X, \tau)$ can be considered as a soft topological space over $X$ with respect to $A=\{a\}$. Consequently, many results from the soft set theory are consequences of the topological results.

Any topological notion can be introduced for a soft topological space by direct reformulation. For example, a soft topological space $(A, X, \tau)$ is soft compact (soft normal, soft Baire, soft metrizable and so on) if the corresponding topological space $\left(A \times X, \tau_{A \times X}\right)$ is compact (normal, Baire, metrizable and so on).

The homeomorphism from Theorem 2.5 is a very good tool for finding a soft topological space which has a soft property $P$ and it has not a soft property $P$. Then, it is sufficient to find a topological space which has the property $P$ and it has not the property $P$.

Recall the notion of an $a$-cluster point from [5]. In parentheses the topological version is given.

Definition 2.4. (see [5]) Let $(A, X, \tau)$ be a soft topological space $((A \times$ $\left.X, \tau_{A \times X}\right)$ be the corresponding topological space) and $a \in A$. A point $x \in X$ is said to be an $a$-cluster point of a soft set $(H, A)$ (of $R_{H} \subset A \times X$ ), if for any soft open set $(G, A)$ (open set $\left.R_{G} \subset A \times X\right)$ such that $x \in_{a}(G, A)\left([a, x] \in R_{G}\right)$, the intersection of $(H, A)$ and $(G, A)$ is not null soft set $\left(R_{H} \cap R_{G} \neq \emptyset\right)$. The set of all $a$-cluster points of $(H, A)$ is denoted by $\operatorname{cl}(H, a)$.

That means, the set $c l(H, a)$ is the set of all points $x \in X$ such that $[a, x]$ is from the closure $c l\left(R_{H}\right)$ of $R_{H}$ in the topological space $\left(A \times X, \tau_{A \times X}\right)$, i.e., $\operatorname{cl}(H, a)=\left\{x \in X:[a, x] \in \operatorname{cl}\left(R_{H}\right)\right\}=\operatorname{cl}\left(R_{H}\right)[a]$. So, the soft closure $c l(H, A)$ of $(H, A)$ (see [5, Proposition 2.14]) is represented by the multifunction $F_{H, A}$ : 
$A \rightarrow X$ defined as follows: $F_{H, A}(a)=\operatorname{cl}(H, a)=\operatorname{cl}\left(R_{H}\right)[a], a \in A$ and its graph is equal to $\operatorname{cl}\left(R_{H}\right)$. Similar connection holds for the soft interior $\operatorname{int}(H, A)$ of $(H, A)$ (see [4, Definition 3.11]).

The next lemma shows a connection between the closure, the interior operator in $\left(A \times X, \tau_{A \times X}\right)$ and the soft closure, the soft interior operator in $(A, X, \tau)$, respectively.

Lemma 2.1. Let $(A, X, \tau)$ be a soft topological space and $\left(A \times X, \tau_{A \times X}\right)$ be the corresponding topological space. Then $\operatorname{cl}(H, A)=\left(F_{c l\left(R_{H}\right)}, A\right), \operatorname{int}(H, A)=$ $\left(F_{\text {int }\left(R_{H}\right)}, A\right)$ and $\operatorname{cl}\left(R_{H}\right)[a]=\operatorname{cl}(H, a), \operatorname{int}\left(R_{H}\right)[a]=\operatorname{int}(H, a)$ for any $a \in A$.

\section{Regularity and $\theta$-topology}

Recall a topological space is $T_{3}$ (regular), if for any closed set $A$ and any point $x \notin A$ their are two disjoint open sets $G, H$ such that $A \subset G$ and $x \in H$. The next characterization is trivial.

Proposition 3.1. A topological space $(X, \tau)$ is $T_{3}$ if and only if for any open set $G$ and any $x \in G$ there is an open set $H$ containing $x$ such that $\operatorname{cl}(H) \subset G$.

Definition 3.1. By [5], a soft topological space $(A, X, \tau)$ is called soft $T_{3}$ if for every point $x \in X$, for every $a \in A$ and for every soft closed set $(Q, A)$ such that $x \notin_{a}(Q, A)$ there exist two soft open sets $(G, A)$ and $(H, A)$ such that $x \in_{a}(G, A), Q(a) \subset H(a)$, and $G(a) \cap H(a)=\emptyset\left(x \notin_{a}(G, A)\left(x \in_{a}(G, A)\right)\right.$ means that $x \notin G(a)(x \in G(a)))$.

As the next example shows their is a soft topological space $(A, X, \tau)$ which is soft $T_{3}$ and the corresponding topological space $\left(A \times X, \tau_{A \times X}\right)$ is not $T_{3}$. Consequently, the topological axiom $T_{3}$ and the soft topological axiom $T_{3}$ are not equivalent.

Example 3.1. Let $A=\{1,2, \ldots\}, X=\left\{x_{1}, x_{2}\right\}\left(x_{1} \neq x_{2}\right)$ and $\tau_{A \times X}$ be the cofinite topology on $A \times X$. It is clear, $\left(A \times X, \tau_{A \times X}\right)$ is not $T_{3}$ (the intersection of any two nonempty open sets is nonempty). Let $\tau=\left\{F_{S}\right.$ : $\left.S \in \tau_{A \times X}\right\}$. Then $(A, X, \tau)$ is a soft topological space, which is soft $T_{3}$. If $x_{1} \notin_{a}(Q, A)$ (the case $x_{2} \notin_{a}(Q, A)$ is similar) and $(Q, A)$ is soft closed, then 
$x_{1} \notin Q(a)$ and $Q(a)=\emptyset$ or $Q(a)=\left\{x_{2}\right\}$. Define $(G, A),(H, A)$ as follows: $G\left(a_{1}\right)=\left\{x_{1}\right\}$ if $a_{1}=a, G\left(a_{1}\right)=X$ if $a_{1} \neq a$ and $H\left(a_{1}\right)=\left\{x_{2}\right\}$ if $a_{1}=a$ and $H\left(a_{1}\right)=X$ if $a_{1} \neq a$. It is clear, $x_{1} \in_{a}(G, A), Q(a) \subset H(a), G(a) \cap H(a)=\emptyset$ and $(G, A),(H, A)$ are soft open.

If we change the set $(Q, A),(G, A),(H, A)$ from Definition 3.1 by the corresponding subsets $S:=R_{Q}, S_{1}:=R_{G}, S_{2}:=R_{H}$ of $A \times X$ respectively, we obtain a topological characterization of the soft $T_{3}$ separation axiom.

Theorem 3.1. A soft topological space $(A, X, \tau)$ is $T_{3}$, if and only if for any $[a, x] \in A \times X$ and any closed subset $S$ of the topological space $\left(A \times X, \tau_{A \times X}\right)$ such that $[a, x] \notin S$ there are two open subsets $S_{1}$ and $S_{2}$ of $\left(A \times X, \tau_{A \times X}\right)$ such that $[a, x] \in S_{1}, S[a] \subset S_{2}[a]$ and $S_{1}[a] \cap S_{2}[a]=\emptyset$.

The set $R_{Q}[a]=S[a]$ is closed and $R_{G}[a]=S_{1}[a], R_{H}[a]=S_{2}[a]$ are open in $\left(X, \tau_{a}\right)$, where $\tau_{a}$ is the induced topology on $X$, then if a soft topological space is $T_{3}$, then the topological space $\left(X, \tau_{a}\right)$ is $T_{3}$ for any $a \in A$. By Proposition 3.1 , we have the next theorem, consequently the converse of Proposition 3.11 of [5] holds.

Theorem 3.2. Let $(A, X, \tau)$ be a soft topological space and $\left(A, X, \tau_{A \times X}\right)$ be the corresponding topological space. Then the following conditions are equivalent.

(3) for any $a \in A$, the topological space $\left(X, \tau_{a}\right)$ is $T_{3}$,

(4) $(A, X, \tau)$ is soft $T_{3}$ (in the sense of Definition 3.1),

(5) for every $x \in X$, for every $a \in A$, and for every a-soft open neighborhood $(F, A)$ of $x$ there exists an $a$-soft open neighborhood $(G, A)$ of $x$ such that $x \in G(a) \subset \operatorname{cl}(G, a) \subset F(a)$ (see Proposition 3.11 of [5]).

Definition 3.2. ([3]) Let $(X, \tau)$ be a topological space. A point $x$ is a $\theta$-interior point of a set $A$, if there is an open set $G$ containing $x$ such that $\operatorname{cl}(G) \subset A$. The set of all $\theta$-interior points of $A$ is denoted by $i_{n}(A)$ and $A$ is called $\theta$-open if any point of $A$ is its $\theta$-interior point, i.e., $A=i n t_{\theta}(A)$. The set of all $\theta$-open sets is denoted by $\tau_{\theta}$.

A point $x$ is a $\theta$-cluster point of $A$, if for any open set $G$ containing $x$, the intersection $A \cap \operatorname{cl}(G)$ is nonempty. The set of all $\theta$-cluster points of $A$ is denoted by $c l_{\theta}(A)$ and $A$ is called $\theta$-closed, if $A=c l_{\theta} A$. 
Remark 3.1. It is clear, $\operatorname{int}_{\theta}(A) \subset \operatorname{int}(A)$ and $\operatorname{int}_{\theta}(A)$ is open. Consequently, $\tau_{\theta} \subset \tau$. Similarly, $\operatorname{cl}(A) \subset \operatorname{cl}_{\theta}(S)$ and $\operatorname{cl}_{\theta}(A)$ is closed.

Proposition 3.2. ([3]) A family $\tau_{\theta}$ of all $\theta$-open sets in a topological space $(X, \tau)$ forms a topology.

The next proposition characterizes the equality $\tau=\tau_{\theta}$.

Proposition 3.3. A topological space $(X, \tau)$ is $T_{3}$ if and only if $\tau=\tau_{\theta}$.

Proof. " $\Rightarrow$ " Let $x \in G \in \tau$. Since $X$ is $T_{3}$, by Proposition 3.1, there is an open set $H$ containing $x$ and $\operatorname{cl}(H) \subset G$, so $G \in \tau_{\theta}$. The opposite inclusion follows from Theorem 3.1.

The implication " $\Leftarrow "$ is clear.

In [5, Definition 5.10 and Definition 5.1], for a soft topological space $(A, X, \tau)$, the set $c_{\theta}(G, a)$ of all $a-\theta$-cluster points and the set $\operatorname{int}_{\theta}(G, a)$ of all $a-\theta$-interior points of a soft set $(G, A)$ were introduced. In fact a point $x \in X$ is an $a-\theta$ cluster point (an $a$ - $\theta$-interior point) of a soft set $(G, A)$ if and only if $[a, x]$ is a $\theta$-cluster ( $\theta$-interior) point of $R_{G}$ in the corresponding topological space $\left(A \times X, \tau_{A \times X}\right)$.

So, for the $\theta$-closure $c l_{\theta}(G, A)$ of $(G, A)$ ([5], Definition 5.11]), the $\theta$-interior $\operatorname{int}_{\theta}(G, A)$ of $(G, A)$ ([5], Definition 5.2) and for the closure $c l_{\theta}\left(R_{G}\right)$ of $R_{G}$, the interior $\operatorname{int}_{\theta}\left(R_{G}\right)$ of $R_{G}$ in $\left(A \times X, \tau_{A \times X}\right)$ the next lemma holds.

Lemma 3.1. Let $(A, X, \tau)$ be a soft topological space and $\left(A \times X, \tau_{A \times X}\right)$ be the corresponding topological space. Then

(1) $\operatorname{cl}_{\theta}(G, A)=\left(F_{c l_{\theta}\left(R_{G}\right)}, A\right), \operatorname{cl}_{\theta}\left(R_{G}\right)[a]=c l_{\theta}(G, a)$,

(2) $\operatorname{int}_{\theta}(G, A)=\left(F_{\text {int }_{\theta}\left(R_{G}\right)}, A\right), \operatorname{int}_{\theta}\left(R_{G}\right)[a]=\operatorname{int}_{\theta}(G, a)$,

By [5], a soft set $(G, A)$ is called $\theta$-open if $\operatorname{int}_{\theta}(G, A)=(G, A)$. By Lemma 3.1 item $(2),(G, A)$ is $\theta$-open if and only if $\left(F_{\text {int }_{\theta}\left(R_{G}\right)}, A\right)=\operatorname{int}_{\theta}(G, A)=(G, A)$ $\Leftrightarrow F_{\text {int }_{\theta}\left(R_{G}\right)}=G \Leftrightarrow \operatorname{int}_{\theta}\left(R_{G}\right)=R_{G}$. So we have the next lemma.

Lemma 3.2. Let $(A, X, \tau)$ be a soft topological space and $\left(A \times X, \tau_{A \times X}\right)$ be the corresponding topological space. Then $(G, A)$ is $\theta$-open (closed) if and only if $R_{G}$ is $\theta$-open (closed) in the corresponding topological space $\left(A \times X, \tau_{A \times X}\right)$. 
As a consequence we have the next theorem which holds in any topological space as well as in any soft topological space.

Theorem 3.3. The sequence $\tau, \tau_{\theta},\left(\tau_{\theta}\right)_{\theta},\left(\left(\tau_{\theta}\right)_{\theta}\right)_{\theta}, \ldots$ is eventually constant if one of them forms a $T_{3}$-space.

\section{Examples}

The converse of Propositions 3.16 and 3.17 in [5] does not hold, as the next two examples show (for definition of soft $e$-continuity and soft $e-\theta$-continuity see $[5])$.

Example 4.1. Let $A=X=(-\infty, \infty)$ and $\tau$ is the usual topology in $A \times X$. Then $(A, X, \tau)$ is a soft topological space $((G, A)$ is soft open if the graph $G r(G)$ of $G$ is open in $A \times X)$. Let $(F, A)$ be given by $F(a)=\{1\}$ for $a \in(0, \infty)$ and $F(a)=\emptyset$ for $a \in(-\infty, 0]$. Then $\operatorname{cl}(F, A)(a)=\{1\}$ for $a \in[0, \infty)$ and $\operatorname{cl}(F, A)(a)=\emptyset$ for $a \in(-\infty, 0)$. So $1 \in_{0} c l(F, A)$ but there is no net $S=\left\{x_{\lambda}, \lambda \in \Lambda\right\}$ of $X$ such that $x_{\lambda} \in_{0}(F, A)$ for any $\lambda \in \Lambda(F(0)=\emptyset)$.

Example 4.2. Let $X=\{x, y\}, A=B=\{1,2\}, F(1)=\{y\}, F(2)=$ $\{x\}, G(1)=\{x\}, G(2)=\emptyset, H(1)=\{x, y\}, H(2)=\{x\}, \tau_{X}=\left\{\mathbf{0}_{A}, \mathbf{1}_{A}\right.$, $(F, A),(G, A),(H, A)\}, Y=\{z, w\}, \tau_{Y}$ be the soft discrete topology (any soft set is soft open). Then $\left(A, X, \tau_{X}\right)$ and $\left(B, Y, \tau_{Y}\right)$ are the soft topological spaces. Let $f(x)=w, f(y)=z, e(1)=1, e(2)=2$ and $f: X \rightarrow Y$ is not soft $e$ continuous at $x$ but the net $S=\left\{x_{\lambda}=x, \lambda \in \Lambda\right\}$ of $X$ soft converges to $x$ and the net $\left\{f\left(x_{\lambda}\right)=w, \lambda \in \Lambda\right\}$ of $Y$ soft converges to $f(x)=w$.

Example 5.27 in [5] is not correct, because $f$ is soft $e$-continuous. A topology $\tau_{2}$ is indiscrete, so $\Phi_{e f}^{-1}\left(\mathbf{1}_{A}\right)=\mathbf{1}_{A}$ and $\Phi_{e f}^{-1}\left(\mathbf{0}_{A}\right)=\mathbf{0}_{A}$. The correct example is the next one.

Example 4.3. Let $X=Y=\{a, b\}, A=B=\{c\}, \tau_{X}=\left\{\mathbf{0}_{A}, \mathbf{1}_{A},(G, A)\right\}$, where $G(c)=\{a\}, \tau_{Y}=\left\{\mathbf{0}_{B}, \mathbf{1}_{B},(H, B)\right\}$, where $H(c)=\{b\}, f(a)=a, f(b)=$ $b, e(c)=c$. Then $\left(A, X, \tau_{X}\right)$ and $\left(B, Y, \tau_{Y}\right)$ are the soft topological spaces. If $c l_{X}$ and $c l_{Y}$ are closure operators in the topological spaces $\left(X, \tau_{c}\right)$ and $\left(Y, \tau_{c}\right)$, then $c l_{X}(\{a\})=c l_{Y}(\{b\})=\{a, b\}, c l_{X}(\{b\})=\{b\}, c l_{Y}(\{a\})=\{a\}$. It is clear, $f$ is soft $e-\theta$-continuous but it is not soft $e$-continuous. 
Example 4.3 also shows that the converse of Proposition 5.33 in [5] does not holds. For $(H, B) \in \tau_{Y}, c l_{\theta}(H, B)=\mathbf{1}_{B}$, so we have $\Phi_{e f}^{-1}((H, B)) \subset$ $\mathbf{1}_{A}=\operatorname{int}_{\theta}\left(\Phi_{e f}^{-1}\left(c l_{\theta}(H, B)\right)\right)$. For $(H, B)=\mathbf{0}_{B},\left((H, B)=\mathbf{1}_{B}\right)$ the inclusion $\Phi_{e f}^{-1}((H, B)) \subset \operatorname{int}_{\theta}\left(\Phi_{e f}^{-1}\left(c l_{\theta}(H, B)\right)\right)$ is evident.

\section{Conclusion}

The main result of the paper is to study a soft topological space by a homeomorphic connection with a topological space on the Cartesian product of two sets. Using this homeomorphic, many soft topological notions can be studied in the corresponding topological space. The paper answers on some questions stated in [5] concerning some soft properties $(\theta$-topology, soft $e$-continuity, soft $e$ - $\theta$-continuity, soft $T_{3}$-separation axiom). The last question from [5] (find a soft topological space such that the sequence $\tau, \tau_{\theta},\left(\tau_{\theta}\right)_{\theta},\left(\left(\tau_{\theta}\right)_{\theta}\right)_{\theta}, \ldots$ is strictly decreasing) is still open.

\section{References}

[1] A. Aygünoğlu, H. Aygün, Some notes on soft topological spaces, Neural. Comput. Appl., 21 (2011), 113-119.

[2] N. Çăğman, S. Karataş, S. Enginoglu, Soft topology, Comput. Math. Appl., 62 (2011), 351-358.

[3] M. Caldas, S. Jafari, M. M. Kovár Some properties of $\theta$-open sets, Divulgaciones Matemáticas, 12, No. 2 (2004), 161-169.

[4] D. N. Georgiou, A. C. Megaritis, Soft set theory and topology, Appl. Gen. Topol., 15, no. 1 (2014), 93-109.

[5] D. N. Georgiou, A. C. Megaritis, V. I. Petropoulos, On Soft Topological Spaces, Appl. Math. Inf. Sci., 7, no. 5 (2013), 1889-1901.

[6] S. Hussain, B. Ahmad, Some properties of soft topological spaces, Comput. Math. Appl., 62 (2011), 4058-4067.

[7] W. K. Min, A note on soft topological spaces, Comput. Math. Appl., 62 (2011), 3524-3528.

[8] Nirmala Rebecca Paul, Remarks on soft $\omega$-closed sets in soft topological spaces, Bol. Soc. Paran. Mat., 33 (2015), 181-190. 
[9] M. Shabir, M. Naz, On soft topological spaces, Comput. Math. Appl., 61 (2011), 1786-1799.

[10] S. Yüksel, N. Tozlu, Z. Güzel Ergül, Soft regular generalized closed sets in soft topological spaces, Int. Journal of Math. Analysis, 8 (2014), 355 367.

[11] I. Zorlutuna, M. Akdag, W. K. Min, S. Atmaca, Remarks on soft topological spaces, Annals of Fuzzy Mathematics and Informatics, 3, no. 2 (2012), $171-185$. 
